\title{
ANÁLISE DA APLICAÇÃO DO SOFTWARE SEPIADES PARA UM ACERVO FOTOGRÁFICO
}

\section{ANÁLISIS DE LA APLICACIÓN DE SOFTWARE SEPIADES EN UNA COLECCIÓN FOTOGRÁFICA}

André Zanki Cordenonsi - andrezc@gmail.com

Docente da Universidade Federal de Santa Maria.

Daniel Flores - flores@smail.ufsm.br

Docente da Universidade Federal de Santa Maria.

Rogério Rocha Ferreira - rogeomatico@yahoo.com.br Mestrando em Patrimônio Cultural pela Universidade Federal de Santa Maria.

\section{Resumo}

Este trabalho propõe a aplicação do software SEPIADES (Safeguarding European Photographic Images for Access Data Element Set) no acervo fotográfico do Avenida Tênis Clube (ATC) visando a instrumentalização de um mecanismo de busca e acondicionamento apropriado para as fotografias digitais e analógicas do acervo fotográfico do ATC. Com a utilização de procedimentos técnicos, a pesquisa de estudo de caso foi utilizada, pois envolveu o estudo profundo e exaustivo de objetos como fotografias e o software SEPIADES, permitindo o seu amplo e detalhado conhecimento. Isso exigiu uma revisão de conceitos e teorias arquivísticas, o entendimento da entidade, sua história, estrutura e funcionamento, assim como, compreender o funcionamento do software, seus pontos positivos e negativos, dos quais pode-se citar: (a) pontos positivos: open source (software livre), facilidade de se observar a hierarquia, descrição multinível, diversidade dos campos para descrição, interface gráfica de fácil compreensão pelo usuário final e gravação dos dados automaticamente; (b) pontos negativos: data e hora do registro dos dados, campo status (aprovado ou não aprovado), OAl (Open Archives Initiative) encontra-se em fase experimental, search (busca), imagem física (imagem thumbnail) de difícil visualização para detalhes da fotografia e ausência da opção de impressão. 
Palavras-chave: Acervo fotográfico. Gestão eletrônica de fotografias. Software SEPIADE.

\section{INTRODUÇÃO}

O objetivo da pesquisa é analisar e desenvolver uma solução tecnológica para o acervo fotográfico do Avenida Tênis Clube (ATC), mais especificamente para a coleção fotográfica dos ex-presidentes do clube. A iniciativa de analisar e desenvolver uma solução tecnológica para o acervo fotográfico surgiu de experiências vivenciadas dentro da entidade Avenida Tênis Clube, em que a busca por fotografias está desperdiçando muito tempo na. O processo de um instrumento de pesquisa tecnológico, ou seja, um software possibilitará uma maior agilidade na busca das fotografias.

\section{ESTUDO DE CASO: AVENIDA TÊNIS CLUBE}

Na década de 1930, foi adotada, por razões ainda não esclarecidas, a data de 7 de setembro de 1916 para a fundação do Avenida Tênis Clube.

No palacete do Dr. Astrogildo de Azevedo, foi realizada a assembléia de eleição da primeira diretoria e todas as demais assembléias e reuniões, até 1920. A primeira assembléia, em 18 de julho de 1917, foi registrada na "Acta I da sessão extraordinária de Assembléia Geral da Sociedade Sportiva Avenida Tennis Club", tendo por objetivo a eleição da diretoria da nova agremiação. Foram eleitas Stellita Campos, presidente, e Aracy Azevedo, vice-presidente.

O Clube possui uma diretoria que corresponde ao Conselho Executivo e ao Conselho Deliberativo. Com relação à sua estrutura física, abrange uma área de, aproximadamente, $36.000 \mathrm{~m}^{2}$, disposta nos seguintes departamentos: recreação, judô, sauna, tênis, tradicionalista, futebol, basquete, bocha e social.

\section{REVISÃo DE LITERATURA}

\subsection{Arquivo, fundo documental, espécie documental e fotografia}

A produção, formal ou mesmo informal, de informações orgânicas registradas origina os arquivos.

Consideram-se arquivos, para fins desta Lei, os conjuntos de documentos produzidos e recebidos por órgãos públicos, instituições de caráter público 
e entidades privadas, em decorrência do exercício de atividades específicas, bem como por pessoa física, qualquer que seja o suporte da informação ou a natureza dos documentos (BRASIL, 1991).

Modernamente, os arquivos deixaram de ser apenas depósitos de documentos e se transformaram em centros ativos de informações a serviço dos usuários, servindo às necessidades da história (RICHTER, GARCIA e PENNA, 2004).

Sendo o arquivo um conjunto documental, o Dicionário Brasileiro de Terminologia Arquivística (2005, p. 97) considera o "conjunto de documentos de uma mesma proveniência um fundo documental".

Fundo, portanto, é definido como o conjunto de documentos produzidos e recebidos por entidades físicas e jurídicas no exercício de suas atividades, necessários ao seu funcionamento e desempenho que guardam relação orgânica entre si e que por isso, não devem misturar-se a outros conjuntos documentais que não tenham a mesma origem (CASTANHO et al., 2001).

Conforme Bellotto (2004, p.52) espécie documental "é a configuração que assume um documento de acordo com a disposição e a natureza das informações nele contidas". As espécies documentais mais freqüentes nos fundos variam de acervo para acervo, podendo ser ofícios, atas, memorandos e fotografias, entre outras.

A fotografia é uma espécie documental que pode ter várias dimensões e conter informações muito valiosas para a entidade que a produziu e para toda a sociedade da qual faz parte.

Sendo assim, o Dicionário Brasileiro de Terminologia Arquivística (2005, p. 95) diz que fotografia é uma "imagem produzida pela ação da luz sobre película coberta por emulsão fotossensível, revelada e fixada por meio de reagentes químicos".

O mesmo princípio de luz é usado na fotografia digital, mas em vez de ser transmitida para um filme é captada para um dispositivo CCD (Charge-coupled device) e é transformada em cargas elétricas que são montadas em forma de um arquivo. Trigo (2006, p.166) afirma que "uma imagem digital é formada por pixels, do inglês picture elements, isto é, os elementos formadores da imagem".

\subsection{Armazenagem}

A armazenagem dos documentos no formato digital deve seguir os mesmos procedimentos de quando o formato do suporte é em papel, seguindo as mesmas regras de classificação. 
Alguns fatores que se deve levar em consideração na armazenagem são: volume e estimativa de crescimento dos documentos, segurança dos documentos, características físicas do suporte e do ambiente, tipos de suporte, freqüência de uso e o custo relativo das opções de armazenamento dos documentos.

A armazenagem de documentos digitais possui três métodos, que são: 1) online, que geralmente é usado em documentos correntes; os equipamentos em que eles estão contidos podem ser o disco rígido ou sistemas de rede, entre outros; 2) o método offline requer a intervenção humana para sua consulta; as informações ficam contidas em CD, DVD e fita magnética; 3) por último, o método nearline, onde os documentos em meio removível permanecem acessíveis através de sistemas de rede.

É fortemente recomendado que documentos eletrônicos de vital importância para o órgão, assim como, documentos digitais com prazos longos de guarda dentro do órgão e documentos digitais de valor permanente sejam armazenados online (CASTRO; CASTRO; GASPARIN, 2007. p.103).

\subsection{Descrição}

A descrição é de vital importância quando o objetivo de um trabalho é relacionar a um documento o máximo de informações pertinentes a ele. "Descrição é o processo intelectual de sintetizar elementos formais e conteúdo textual de unidades de arquivamento, adequando-os ao instrumento de pesquisa que se tem em vista produzir (inventário sumário ou analítico, guia, etc.)”. (PAES, 2007, p.25).

O processo de descrição necessita de um trabalho intenso e minucioso em toda a documentação, partindo-se sempre da estrutura como um todo até se chegar ao documento.

Para se fazer descrição, atualmente, existem várias normas que se pode tomar como base, tais como: a NOBRADE (Norma Brasileira de Descrição Arquivística) e a ISAD (G) (Norma Geral Internacional de Descrição Arquivística), entre outras.

Em um trabalho de descrição é de fundamental importância a análise dos documentos item por item, para que nenhuma informação seja deixada de lado, por menos importante que possa parecer no momento de se descrever. 


\section{SEPIADES (Safeguarding European Photographic Images for Access Data Element Set)}

Visando a manter acessíveis as coleções em arquivos e bibliotecas e proporcionando o intercâmbio de conhecimento com conferências, reuniões e workshops, constituiu-se, em 1994, a Comissão Européia de Preservação e Acesso (European Commission on Preservation and Access - ECPA). Com essas experiências, em 1999, o Projeto Europeu de Arquivo Visual (European Visual Archive-project - EVA), no âmbito do programa INFO 2000, realizou um estudo sobre a preservação de coleções fotográficas européias. Como resultado dessa pesquisa, foi observado que eram usados vários modelos descritivos já existentes, além de adaptarem alguns modelos para descrever coleções fotográficas.

A partir daí teve início o Programa Europeu de Salvaguarda de Imagens para Acesso (Safeguarding European Photographic Images for Access - SEPIA) que se destina a definir o papel das novas tecnologias em um contexto de longo prazo de preservação histórica para coleções fotográficas e com a idéia de criar um modelo básico para descrição de fotografias.

A partir de reuniões do grupo de trabalho SEPIA, foi apresentado um relatório consultivo com o modelo SEPIADES. Este é antes de tudo um modelo que pode ser usado para descrever coleções de fotografias. Basicamente, o modelo pode funcionar ao lado de modelos descritivos já existentes, mas é claro que também pode ser implementado como uma ferramenta distinta e independente. O SEPIADES é um software com código aberto, ou seja, open source (software cujo código de programação é aberto para, sob uma licença, ser usado, modificado e redistribuído, com ou sem as alterações nele empreendidas) para a descrição de fotografias e que permite a inclusão de metadados.

Com uma investigação feita em diferentes modelos de descrição multi-nível existentes, como a ISAD (G) (Norma geral internacional de descrição arquivística) e o Fotios (Dutch descriptive model for photographic materials), com a finalidade de manter a aplicabilidade do modelo SEPIADES em coleções já existentes, conclui-se que um modelo de descrição deve ter a capacidade de trabalhar com vários níveis de uma coleção; assim, a estrutura hierárquica é determinada pelo catalogador, permitindo a utilização ilimitada de níveis e subníveis, o que não se pode fazer em alguns modelos multinível. 
O software SEPIADES possui funções de descrição multinível (árvore hierárquica), armazenamento de registros em formato XML (eXtensible Markup Language); função de exportação de acordo com o recomendado pelo mapeamento no relatório consultivo, implementação do Open Archives Initiative Protocol for Metadata Harvesting (OAI-PMH), permitindo que os usuários possam compartilhar seus dados com outras pessoas com o mínimo esforço, e Open Source (código aberto - software livre) programados na linguagem de programação Java e utilizando Java Runtime Environment (JRE) para sua execução. Isso significa que pode ser executado em GNU/Linux, MS Windows ou qualquer outro sistema operacional que possua a JRE, permitindo a integração flexível com pacotes de softwares descritivos existentes.

A árvore hierárquica é uma das principais funções do SEPIADES e possui vários níveis (Figura 1).

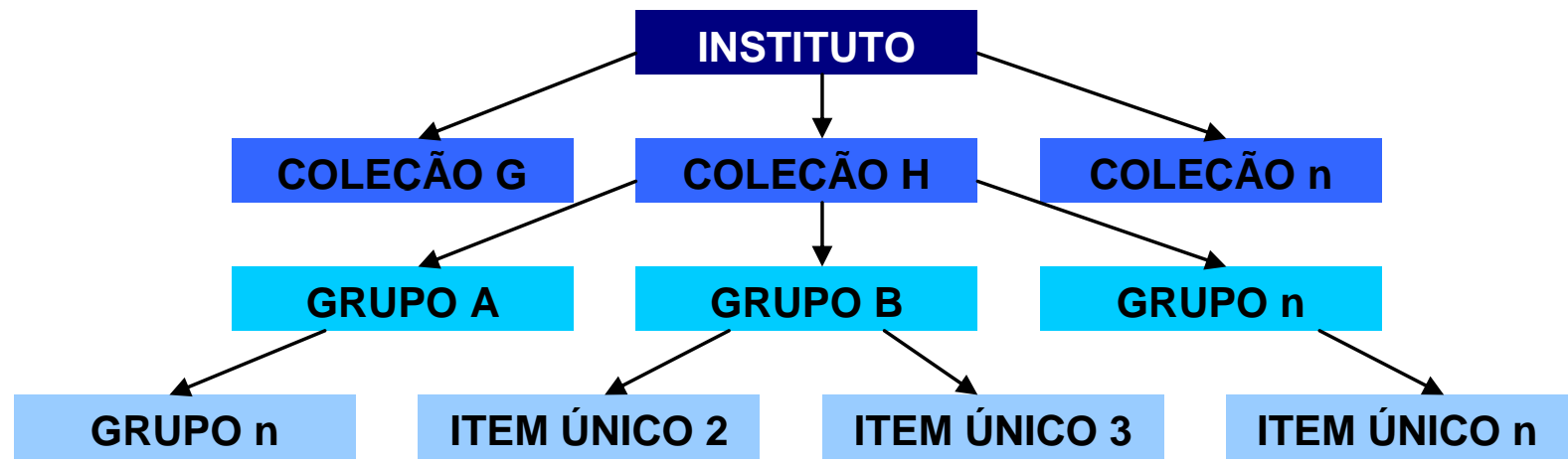

Figura 1 - Estrutura Hierárquica do modelo SEPIADES

O nível mais elevado dessa hierarquia é o instituto, com informações básicas como endereço, país e uma breve descrição do instituto da(s) coleção(ões). Essas informações podem ser relevantes, por exemplo, se são trocadas com outros institutos. Um instituto possui um ou mais conjuntos (coleção, grupo, item).

O próximo nível é o da coleção, que é um grupo de objetos que foram reunidos por um indivíduo ou organização. Cada coleção é constituída por um ou mais agrupamentos, que é o próximo nível. $O$ agrupamento é um agregado de imagens físicas que poderia ser uma subdivisão de uma coleção ou de outro agrupamento.

Cada coleção ou agrupamento é constituído por um ou mais itens (que são únicos), que será o último nível. 


\section{DESENVOLVIMENTO DE UMA COLEÇÃO FOTOGRÁFICA NO SOFTWARE SEPIADES}

Para o estudo do software SEPIADES, o mesmo foi instalado em um computador particular, pois na instituição não havia computador.

O programa foi adquirido na página da web http://www.knaw.nl/ecpa/ sepia/workinggroups/wp5/download.html; também foi feito, na mesma página o download do programa Java Runtime Environment (JRE), que é necessário para o funcionamento do software SEPIADES.

Com o software instalado, pode-se enfim começar o seu estudo. Esse estudo foi basicamente através de seus manuais que são bastante complexos e completos.

A seguir será apresentada, passo-a-passo, a inserção dos dados no software SEPIADES.

Para todos os níveis, exceto para o nível instituto, há três abas, que representam as divisões:

1 Administração - elementos administrativos como: catalogador, data de entrada, etc.;

2 Proveniência - elementos sobre a história e antecedentes do instituto, aquisição, imagem física e imagem visual;

3 Material - elementos sobre o conteúdo de forma física e do material.

A primeira etapa consiste em clicar em "add" para que seja possível colocar o nome da instituição (Avenida Tênis Clube) (Figura 2). 


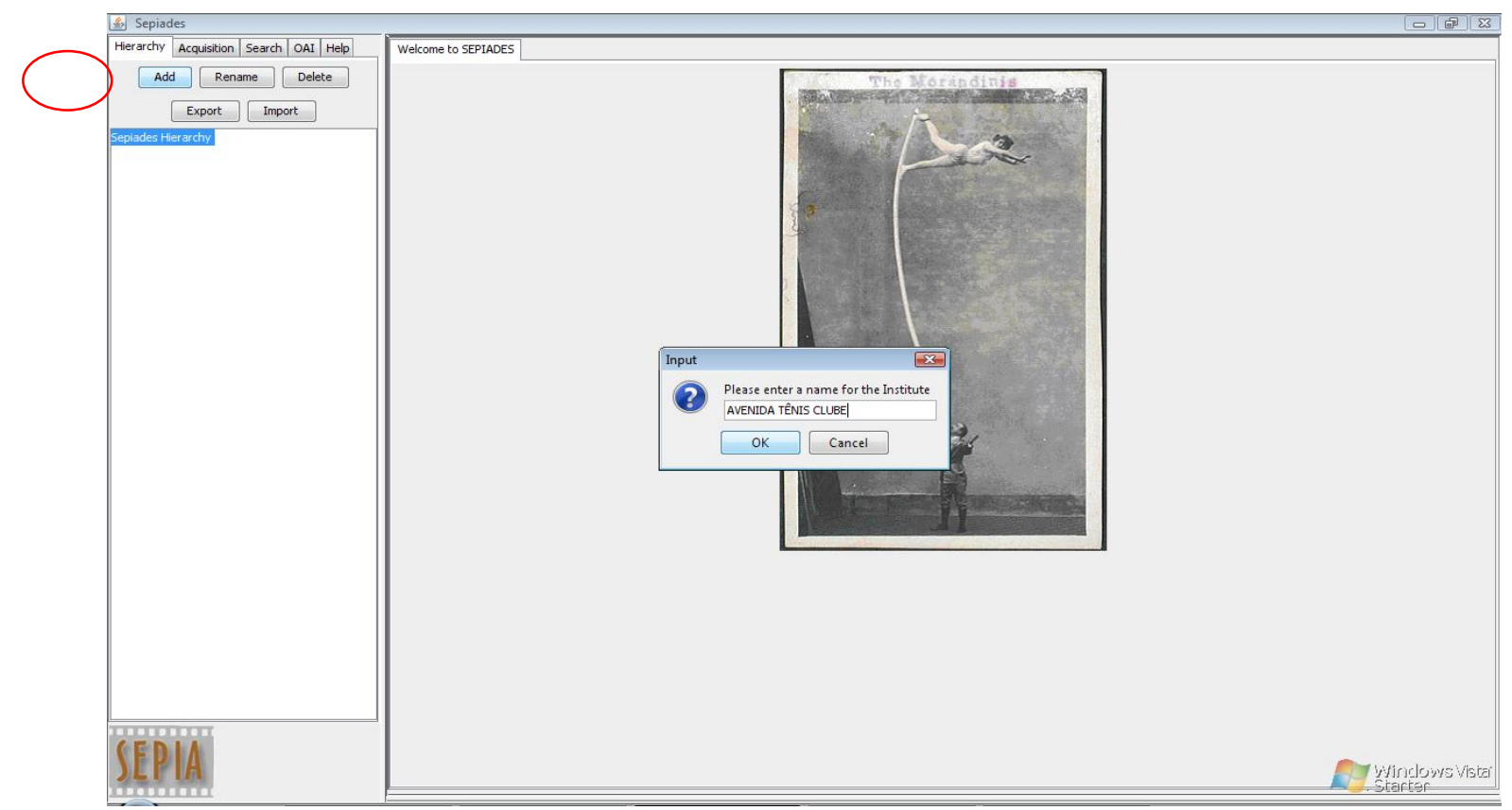

Figura 2 - Instituição

O programa apresenta uma tela para que seja possível colocar as informações referentes à instituição conforme a norma ISO 8601 para o preenchimento dos dados.

O próximo passo será o nível da aquisição, onde se seleciona a aba "acquisition" e, depois, clica-se em "add” (Departamento Administrativo) (Figura 3).

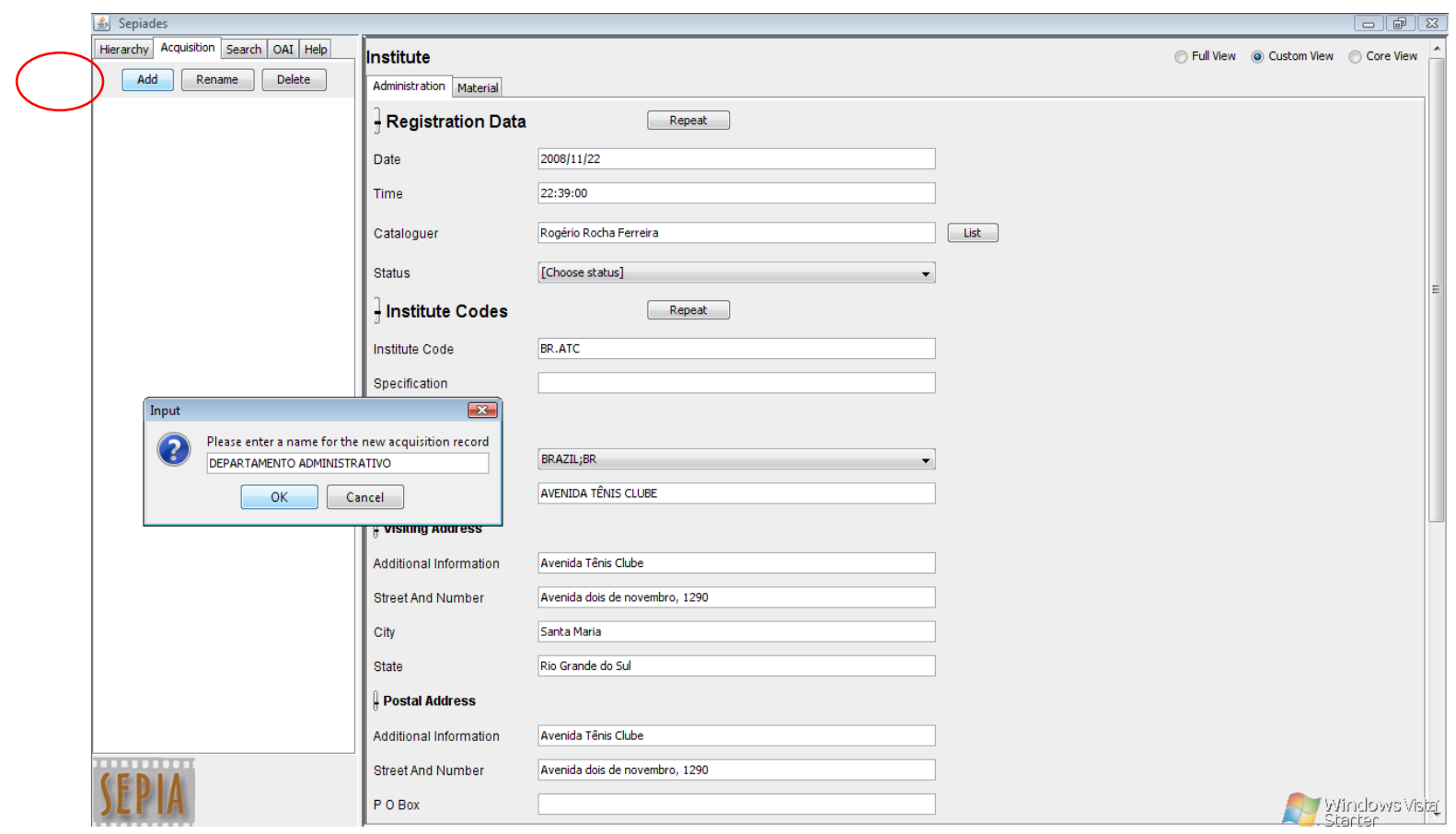

Figura 3 - Aquisição 
$\mathrm{Na}$ próxima tela se deve informar os dados sobre a aquisição (Departamento Administrativo) conforme a ISO 8601.

Selecionando "Hierarchy", clica-se em "add" e, na caixa, seleciona-se a aquisição e "ok" (Figura

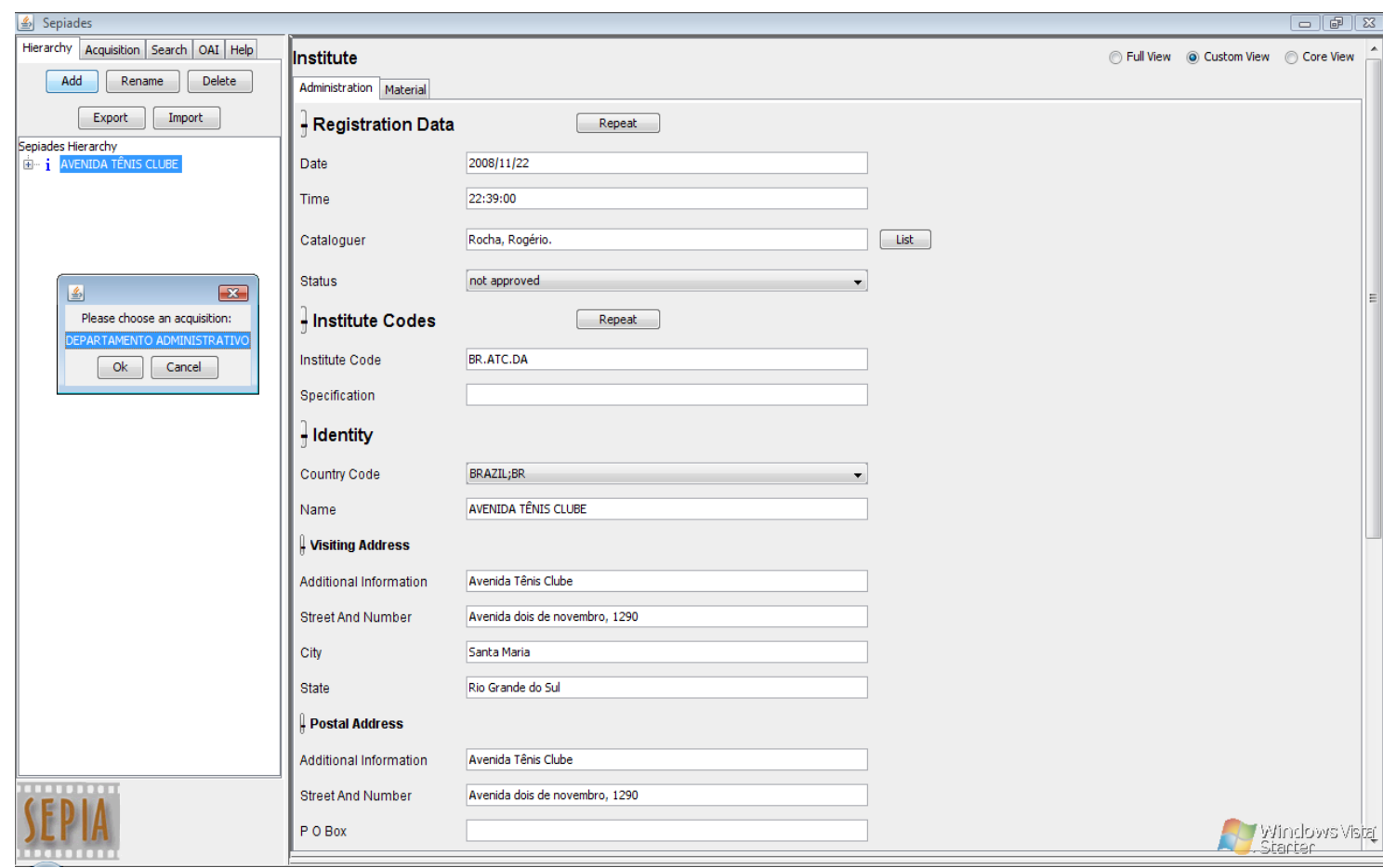

Figura 4 - Adicionar a aquisição na hierarquia

Na próxima tela, será pedido o nome da coleção (Histórico) que será o nível de série/subsérie (Figura 5).

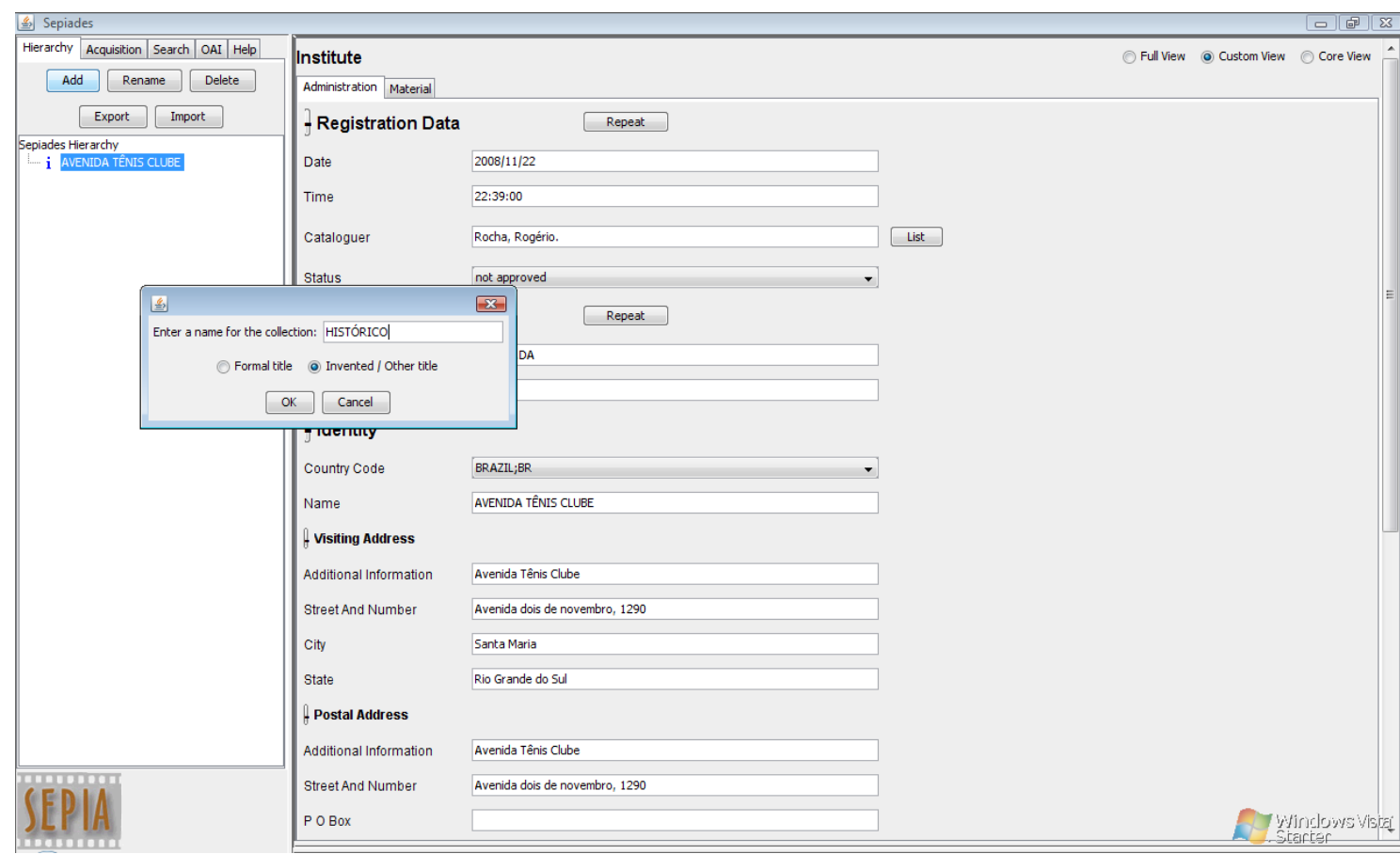


Figura 5 - Adicionar a coleção na aquisição

Na próxima tela se deve informar os dados sobre a coleção (Histórico) conforme a ISO 8601. Para adicionar o nível do grupo/dossiê, primeiro se deve selecionar o nível da coleção (Histórico dos ex-presidentes), clicar em "add" e somente então aparecerá uma janela com as opções "grouping" e "acquisition". Foi selecionado "grouping" e a opção "ok" (Figura 6).

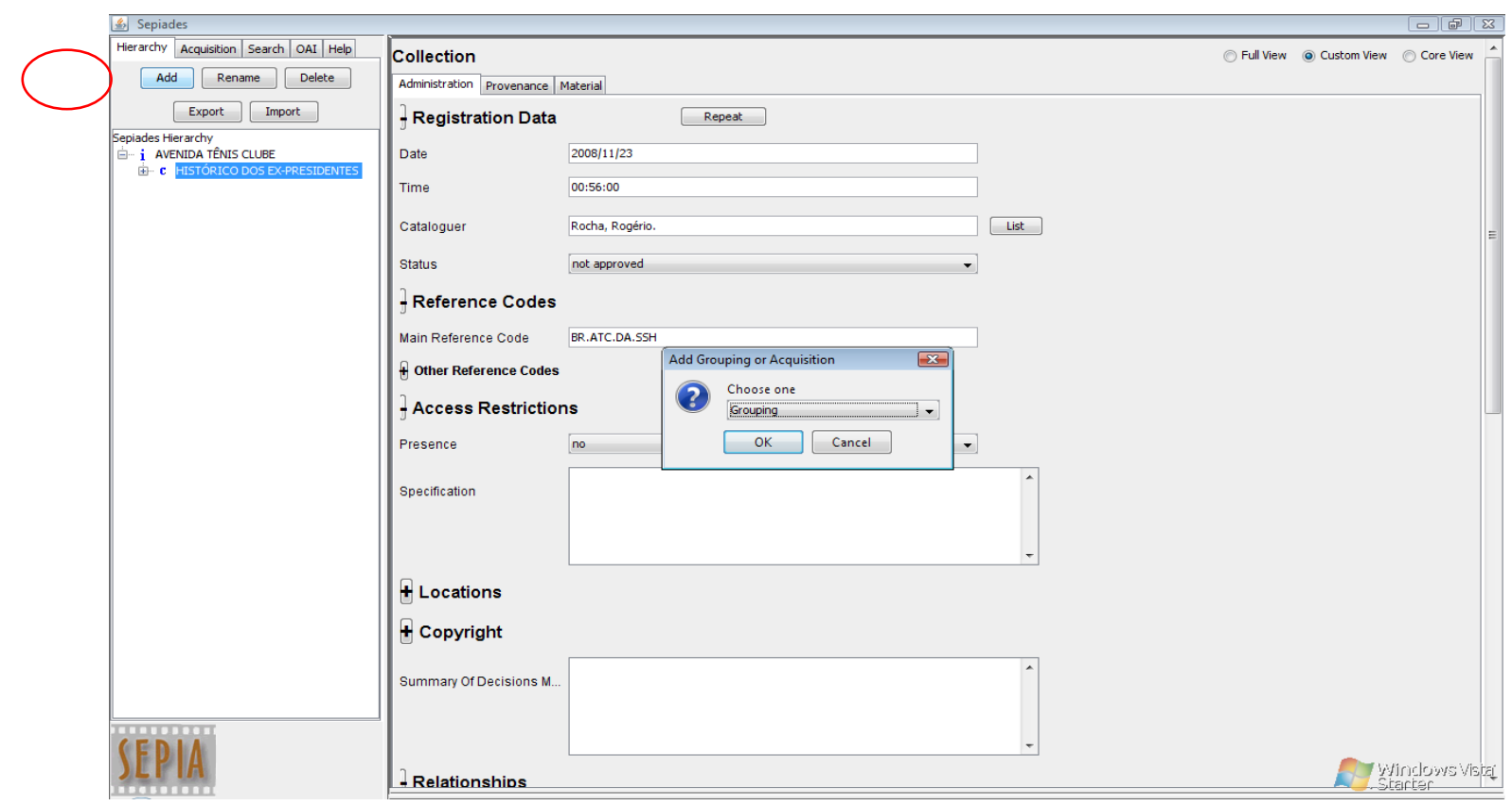

Figura 6 - Nível do grupo/dossiê

O próximo passo será dar nome ao grupo/dossiê (Galeria dos ex-presidentes) na janela que será mostrada.

Depois de inserido o grupo/dossiê (Galeria dos ex-presidentes), deve-se selecionálo na janela, para inserção dos dados referentes a ele (Figura 7). 
Análise da aplicação do software SEPIADES... André Z.Cordenonsi; Daniel Flores; Rogério R. Ferreira

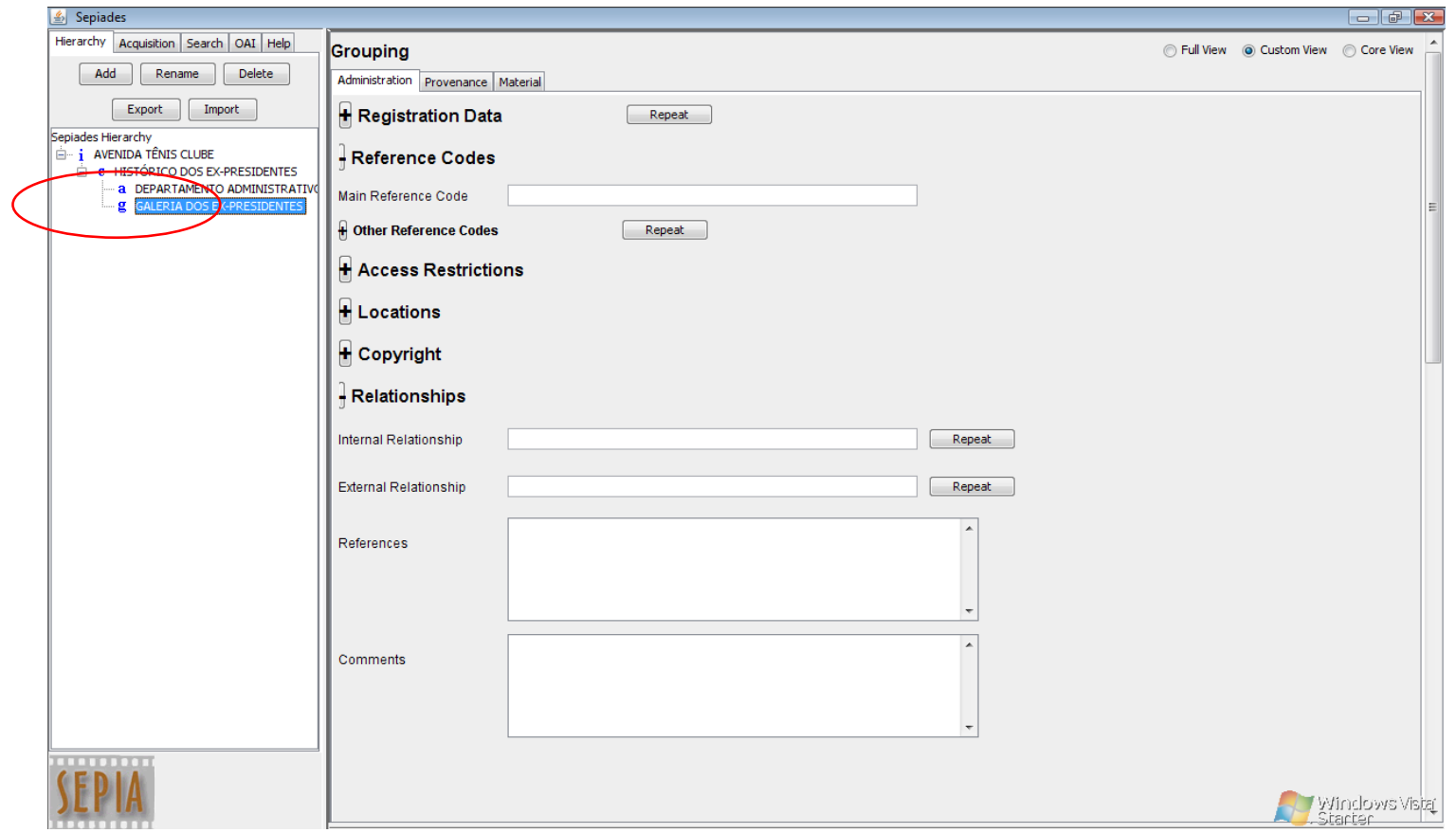

Figura 7 - Inserção dos dados do grupo/dossiê

Após a inserção dos dados, deve-se selecionar o grupo/dossiê (Galeria dos expresidentes), clicar em "add" e somente então aparecerá uma janela com as opções "grouping" e "visual image". Seleciona-se "visual image" e a opção "ok". Esta opção vai inserir na hierarquia o item documental (Figura 8).

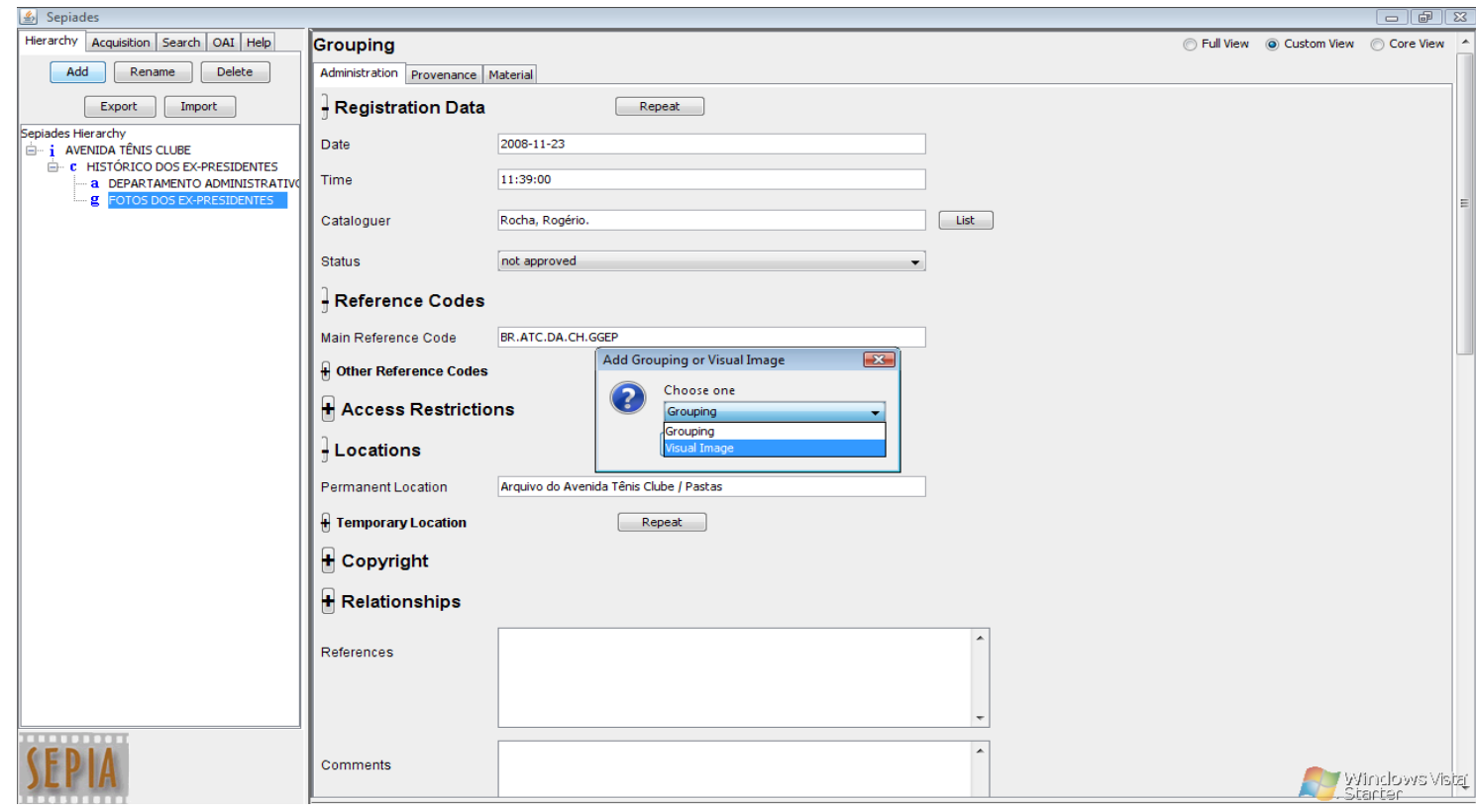

Figura 8 - Nível de item documental 
Depois de selecionado "visual image" na janela anterior, esta opção aparecerá na árvore de hierarquia da próxima janela como "view images". Selecionando-a, aparecerão ao lado opções para inserção de dados referentes a ela (Figura 9).

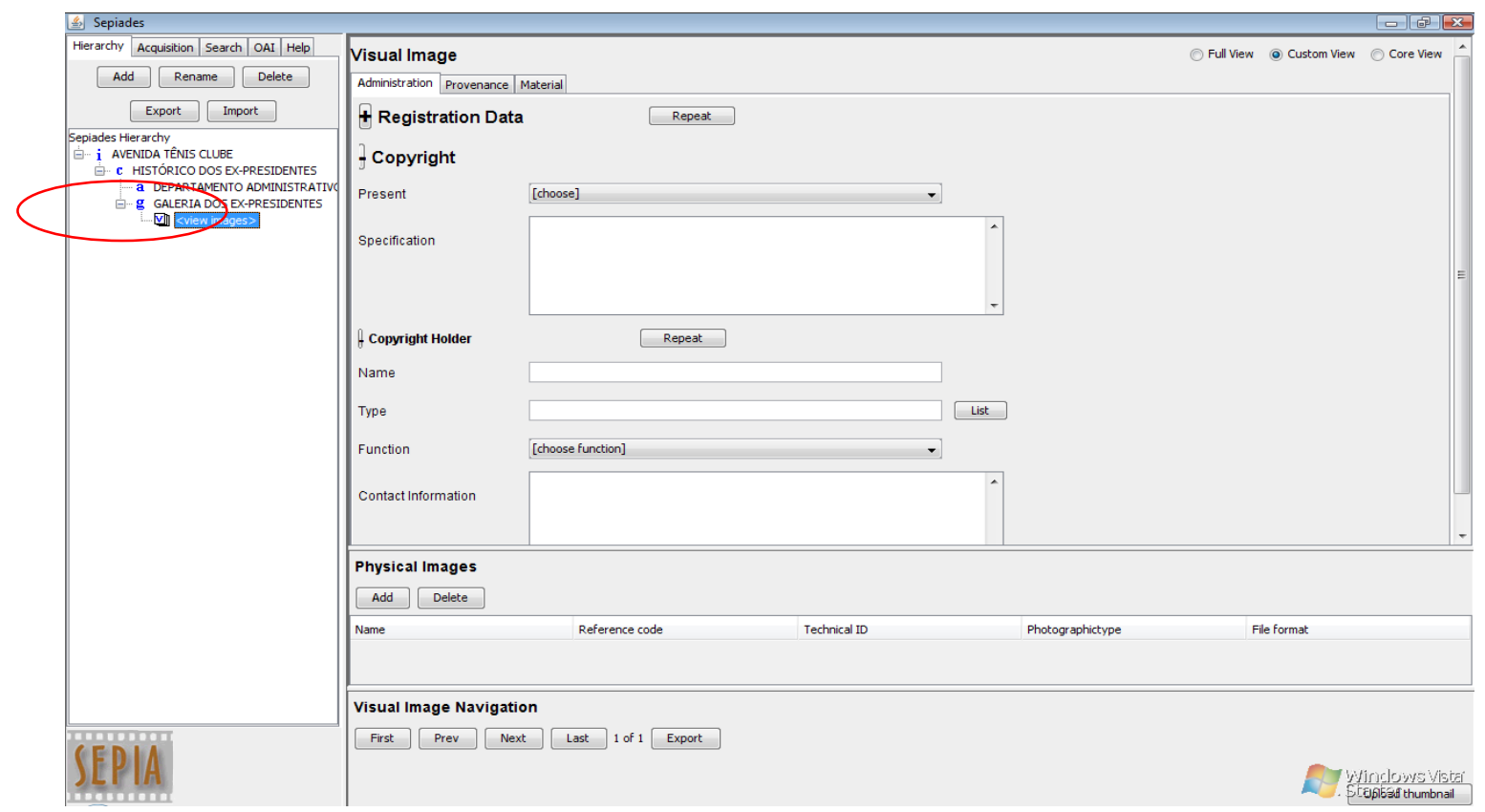

Figura 9 - Inserção dos dados da Imagem Visual

Depois de inseridos os dados em "visual image", deve-se clicar em "add" na parte inferior da janela abaixo de "physical images" para que sejam colocados os dados da imagem física (Figura 10).

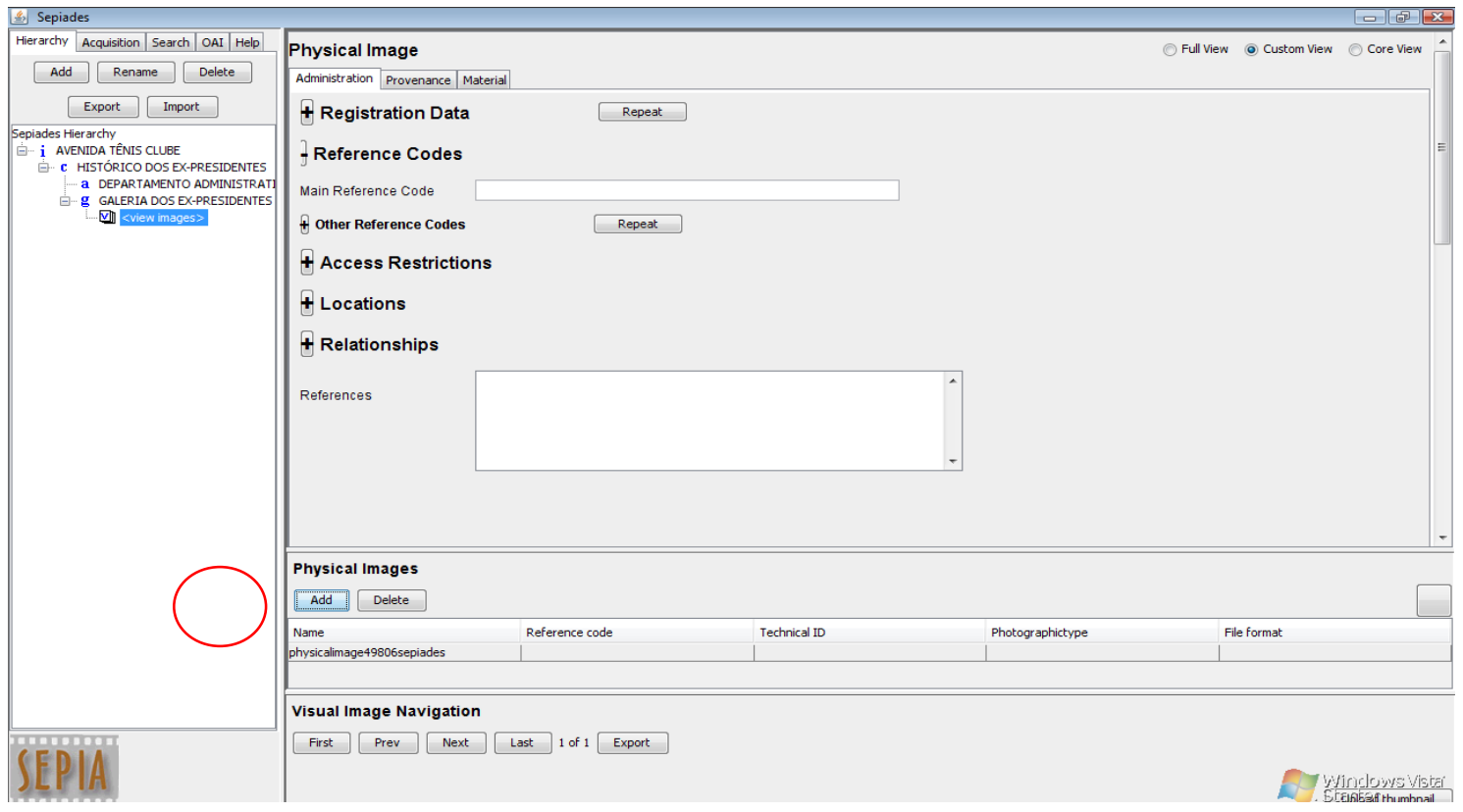

Figura 10 - Inserção dos dados da Imagem Física 
Depois de inseridos os dados da "visual image" e da "physical images", deve-se clicar em "upload thumbnail" no canto inferior direito da janela. Então, através da janela que irá aparecer, seleciona-se a imagem que pertence às informações e se clica em "open" para adicionar a imagem. Com a imagem selecionada, ela aparecerá no canto inferior direito da janela (Figura 11).

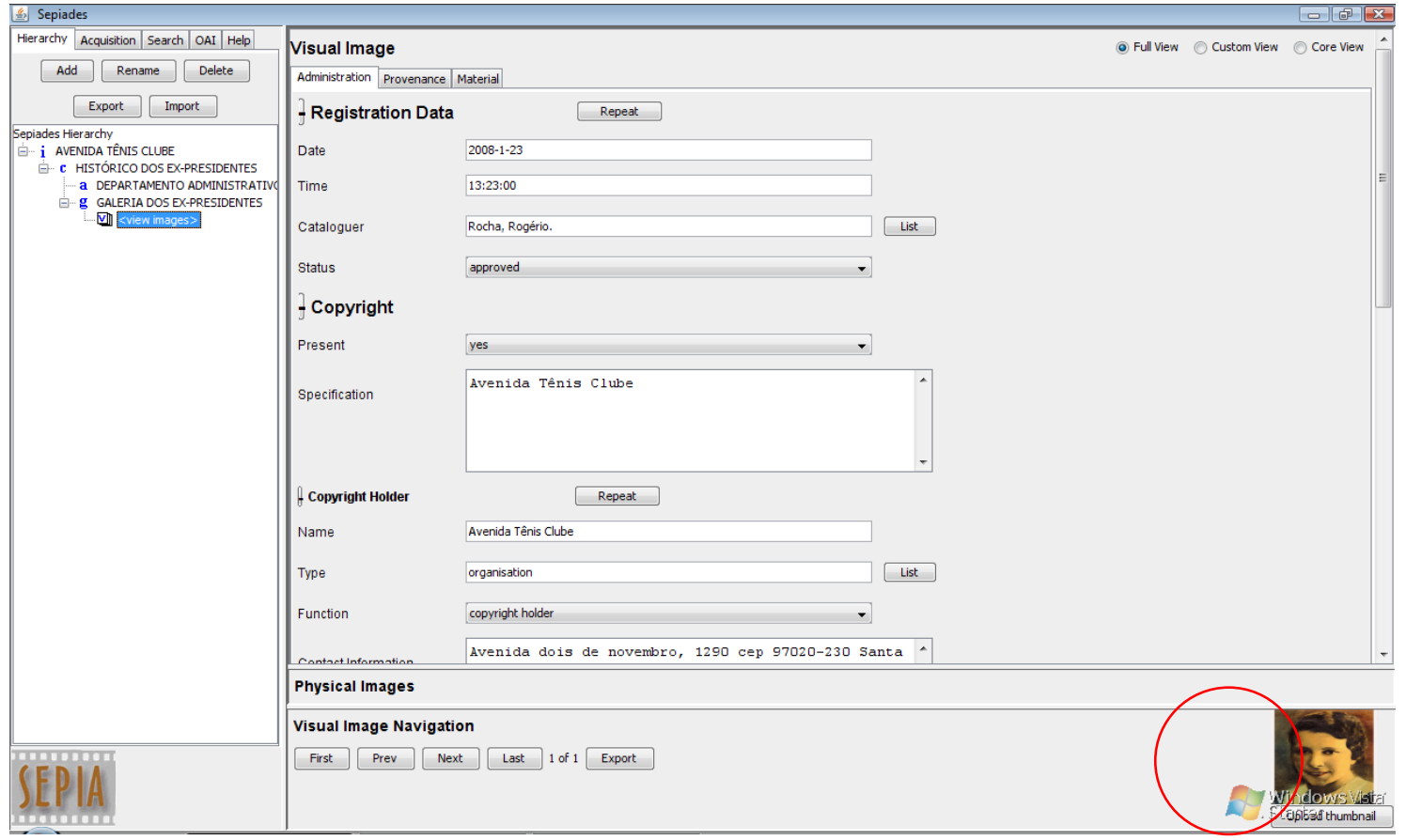

Figura 11 - Imagem inserida

Para retornar a "visual image" basta clicar no ícone com as informações de descrição da imagem, no canto inferior direito da janela. Caso se deseje ir para "physical image", basta selecionar o nome da imagem.

Para consulta, basta selecionar a aba "search" e no campo que aparecer, digitar o que deseja pesquisar. Para compartilhamento, há a opção OAI (Open Archives Initiative), que permite compartilhar dados com outras pessoas com o mínimo esforço. Deve-se selecionar a aba "OAl" e "start server".

Para ajuda, seleciona-se a aba "help" e as informações (em inglês) serão disponibilizadas. Para uma ajuda mais específica, basta clicar na opção desejada e automaticamente aparece uma janela com informações (em inglês) sobre o item selecionado. 


\section{ANÁLISE E CONCLUSÕES}

Para um bom entendimento da situação da organização em que se encontra a instituição foi necessário compreender o seu funcionamento, sua história, estrutura, funções e atividades desenvolvidas. Isso se deu através do levantamento de dados em documentos administrativos, estatutos, regulamentos e no site do clube. Entender uma instituição é tentar inserir-se o máximo possível nas suas rotinas e observar o seu funcionamento, é fazer parte dela em todos os seus setores para analisar e não julgar a sua situação e necessidades.

Com a observação e estudo do acervo, constatou-se que a utilização das fotografias se dá com mais freqüência na realização de relatórios e clippings feitos pelos departamentos, principalmente pelo departamento de divulgação e eventos e pelo departamento de esportes. O setor de relações públicas busca assiduamente por fotografias para serem divulgadas em seus informativos ou em suas promoções de divulgação dos eventos mais diversos.

Como o acervo é muito grande, com aproximadamente sete mil fotografias dos mais variados tamanhos, além de negativos e slides, foi necessário que se delimitasse uma coleção para que a pesquisa fosse realizada.

A coleção com melhores condições de acesso e com maior necessidade de tratamento foi a coleção dos ex-presidentes que, conforme a organização da instituição, está inserida no Departamento Administrativo, Série Organização e Funcionamento junto à Coleção dos ex-presidentes.

Através de pesquisa em atas pode-se constatar lacunas na relação dos expresidentes do ATC. Dos quarenta e nove presidentes encontrados na verificação das atas, trinta e oito fazem parte da galeria dos ex-presidentes, sendo que dos onze restantes, dez não figuravam na galeria e um no momento da pesquisa, era o presidente em exercício. Dos dez ex-presidentes que foram verificados nas atas, foi encontrada, após pesquisa nos dossiês dos sócios, apenas a fotografia de um ex-presidente; de alguns outros, nem a documentação foi encontrada.

Como algumas fotografias não estavam no formato digital, optou-se por digitalizar as mesmas com o mesmo formato das que já haviam sido digitalizadas, sendo o formato de arquivo utilizado o JPEG.

O formato de arquivo JPEG foi estabelecido por ter algumas vantagens, tais como praticamente todas as câmeras digitais salvarem as fotos no formato JPEG e oferecer boa 
qualidade e muita rapidez de processamento. Foi realizada, então, a digitalização das três fotografias que não haviam sido passadas do formato de papel para o digital.

O aparelho utilizado foi uma multifuncional Hewlett-Packard (HP) modelo PSC1315 all-in-one e software HP Image Zone versão 4.2. A resolução da imagem foi de 300 dots per inch (dpi) (pontos por polegada), que está diretamente relacionada à definição da imagem (fotografia), sendo que, quanto maior a resolução, mais pontos teremos por polegada e, consequentemente, maior será a nitidez da imagem.

A análise foi feita ao mesmo tempo em que foi realizada a coleta das informações descritivas das fotografias digitais, já que as informações coletadas em atas em etapa anterior foi em relação à imagem visual, ou seja, informações como: quem, quando, onde e gestão, entre outras.

$\mathrm{Na}$ medida em que as informações descritivas foram sendo digitadas nos campos específicos do software é que se pode perceber que o mesmo é extremamente detalhista; isto se deve à exigência dos diferentes tipos de necessidades e dos diversos tipos de usuários que o software deve atender.

O software apresenta alguns pontos positivos e negativos que foram sendo observados durante a inserção dos dados nos seus respectivos campos:

1 Pontos positivos:

1.1 Open source - software livre, permite sua aquisição sem custo algum;

1.2 Facilidade de se observar a hierarquia - isso facilita quando se tem diversos níveis e subníveis na instituição;

1.3 Descrição multinível - a descrição se dá em todos os níveis da hierarquia: instituto (acervo da entidade), aquisição (fundo), coleção (série ou subsérie), grupo/subgrupos (dossiê/processo) e item único (item documental), este último se dividindo em imagem visual e imagem física.

1.4 Diversidade dos campos para descrição - quanto mais campos apresentados mais informações podem ser disponibilizadas para posterior consulta;

1.5 Interface - apesar de ser um software em outro idioma (inglês), possui fácil compreensão para sua utilização pelo usuário final; 
1.6 Gravação - na medida em que os dados vão sendo digitados, são gravados automaticamente, evitando-se, assim, perda de informações por motivos de troca de abas ou até desligamento acidental do computador.

2 Pontos negativos:

2.1 Data e Hora - a data de registro é inserida manualmente quando deveria ser feita automaticamente para evitar erros;

2.2 Status (aprovado ou não aprovado) - o status aparece sempre no início de todos os níveis como Registration Data, o que indica aprovação da instituição. Esta aprovação é que vai indicar se o mesmo será disponibilizado ao público (as informações). O ideal seria que a mesma fosse colocada por último em todos os níveis, permitindo que a decisão sobre a aprovação ocorresse somente após a inserção final dos dados;

2.3 OAI - encontra-se em fase experimental e deve ser configurado para poder ser utilizado;

2.4 Search (busca) - retorna inúmeros resultados desnecessários;

2.5 Imagem física - a imagem thumbnail apresenta-se muito pequena com dificuldade para observação de detalhes da fotografia. Seria interessante haver uma opção para ampliação da imagem física, caso houvesse necessidade de melhor observação da mesma;

2.6 Impressão - não possui uma opção de impressão dos dados, ou até mesmo de informações junto com a imagem física, que muitas vezes é necessário.

Como o mesmo é um software livre fica mais acessível para que as falhas encontradas sejam corrigidas ainda que o programa seja para descrição.

\section{REFERÊNCIAS}

AASBO, K; GARCÍA, I. O.; ISOMURSU, A; JOHANSSON, T; KLIJN, E. SEPIADES. Recommendations for cataloguing photographic collections: advisory report by the SEPIA Working Group on Descriptive Models for Photographic Collections. (SEPIA). European Commission on Preservation and Access. Amsterdam, 2003.

Disponível em: < http://www.knaw.nl/ecpa/publ/pdf/2710.pdf>. Acesso em: 21 ago. 2008.

BELLOTTO, H. L. Arquivos permanentes: tratamento documental. $2^{\mathrm{a}}$.ed. rev. e ampl. Rio de Janeiro: Editora Fundação Getúlio Vargas. 2004. 
BRASIL. Leis, Decretos, etc. Lei n. 8.159, de 8 de janeiro de 1991. Dispõe sobre a política nacional de arquivos públicos e privados e dá outras providências. Diário Oficial da República Federativa do Brasil, Brasília, DF, n. 6, 9-1-1991. Disponível em: <http://www.planalto.gov.br/CCIVIL /leis/L8159.htm> Acesso em: 02 fev. 2009.

CASTANHO, D. M.; GOMES, D. C.; DOTTO, E. R.; GARCIA, O. M. C.; SILVA, Rosani B. P. Uma política de arranjo documental para a Universidade Federal de Santa Maria. Santa Maria, RS, 2001.

CASTRO, A. M.; CASTRO, A. M.; GASPARIN, D. M. C.. Arquivos: físicos e digitais. Brasília: Thesaurus, 2007. $186 \mathrm{p}$.

DICIONÁRIO Brasileiro de Terminologia Arquivística. Rio de Janeiro, 2005. Disponível em: <www.arquivonacional.gov.br/download/dic_term_arq.pdf>. Acesso em: 20 abr. 2007.

LUSENET, Y. de; KLIJN, E. Cataloguing photographic collections. European Commission on Preservation and Access. Amsterdam. 2004.

Disponível em: < http://www.knaw.nl/ecpa/publ/pdf/2719.pdf >. Acesso em: 21 ago. 2008.

PAES, M. L. Arquivo: teoria e prática. Rio de Janeiro. Editora Fundação Getúlio Vargas, 2007.

RICHTER, E. I. S.; GARCIA, O. M. C.; PENNA, E. F. Introdução à Arquivologia. 2aㅡ. ed. FACOS-UFSM. 2004.

TRIGO, T. Equipamento fotográfico: teoria e prática. São Paulo. Editora Senac SP. $2^{\mathrm{a}}$ ed. rev. e amp. 2003. p.264.

\section{Title}

An analysys about the SEPIADES software application in a photographic collection

\section{Abstract}

This paper proposes the application of software SEPIADES (Safeguarding European Photographic Images for Access Data Element Set) in the Avenida Tênis Clube (ATC) photograph collection to the instrumentalization of a search engine and packaging suitable for the photos digital and analog photographic collection of the ATC. With the use of technical procedures, the investigation of the case study was used because involved the deep and comprehensive study of objects such as photographs and SEPIADES software, allowing its broad and detailed knowledge, this required a review of archival theories and concepts, understanding the entity, its history, structure and functioning, as well as understand the functioning of the software, its strengths and negative, of which we can mention: (a) positive: open source (free software), easy to observe the hierarchy, multilevel description, diversity of fields for description, graphical interface easily understood by the end user and recording of data automatically, (b) negative points: the date and time of registration data and field status (approved or not approved), OAI (Open Archives Initiative) is in the experimental phase search (search), image physical 
(thumbnail image) difficults the visualization of details of the photograph and lack the option of printing.

\section{Keywords}

Photographic Collection. Management Electronic photographs. Software SEPIADES.

\section{Título}

Análisis de la aplicación de software SEPIADES en una colección fotográfica

\section{Resumen}

Este trabajo propone la aplicación de software SEPIADES (Safeguarding European Photographic Images for Access Data Element Set) en la colección fotográfica del Avenida Tênis Clube (ATC) cuyo objetivo es la manipulación de un motor de búsqueda y embalaje adecuado para las líneas analógicas y fotografías digitales de la colección fotográfica de ATC. Con el uso de procedimientos técnicos, estudio de investigación caso se usó porque implicaba el estudio profundo y exhaustivo de objetos tales como fotografías y el software SEPIADES, permitiendo su conocimiento amplio y pormenorizado. Esto requiere una revisión de las teorías y los conceptos de archivo, la comprensión de la entidad, su historia, estructura y funcionamiento, así como entender cómo funciona el software, sus fortalezas y debilidades de los cuales podemos mencionar: (a) los puntos positivos: de código abierto (software libre), fácil de observar la jerarquía, multinível descripción, la diversidad de campos para la descripción, la interfaz gráfica de fácil comprensión por el usuario final y grabar automáticamente los datos, (b) los puntos negativos: la fecha y hora del registro de datos, el estado del campo (aprobados o no aprobados), OAl (Open Archives Initiative) está en desarrollo, de búsqueda (búsqueda), la imagen física (imagen en miniatura) es difícil de ver los detalles de la fotografía y ninguna opción de impresión.

\section{Palabras Clave}

Colección Fotográfica. Gestión electrónica de fotos. Software SEPIADES.

Recebido em: 22.04.2010

Aceito em: 15.09 .2010 